\title{
Piecewise Linear Feedback Rules in a Non Linear Model of the Phillips Curve: Evidence from the US and the UK.
}

\author{
Luisa Corrado \\ University of Cambridge, \\ Department of Applied Economics, UK. \\ Sean Holly \\ University of Cambridge, \\ Department of Applied Economics, UK.
}

November 9, 2000

\begin{abstract}
Optimal nominal interest rates rule are usually set assuming that the underlying world is linear. Our work relaxes this assumption and examines the performance of optimal rules when non-linearities are present. In particular if the inflation-output trade off exhibits non linearities (convexities) this will impart a bias to inflation when a linear rule is used. To correct this bias we propose a piecewise linear rule, which can be thought of as an approximation to the non-linear rule of Schaling (1999). We show that this reduces the bias, but at the expense of an increase in the volatility of the nominal interest rate. Finally we examine how the zero floor on nominal interest rate affects output and inflation when both rules are adopted. With a linear feedback rule the output variability increases since nominal interest rate cannot be further reduced in presence of adverse shocks. The adoption of a piecewise rule with a zero floor on interest rates successfully reduces output volatility. Significant differences in the transmission mechanism of monetary policy, between the USA and the UK, show up both in the form of the optimal feedback rule and in the distribution of outcomes when there is a zero floor to nominal interest rates and non-linearities in the Phillips curve.
\end{abstract}

\section{Introduction}

The increasingly widespread use of institutionally independent bodies to operate monetary policy and the use of the nominal short term interest rate as the instrument has stimulated considerable research into how interest rates should be set in a stochastic environment. They can be either rules à la Taylor where nominal interest rates respond to current deviations of inflation and output from target; or as Svensson (1997) has recently suggested, a two year ahead inflation forecast should be targeted, since there is an inherent lag in setting the interest rate and it showing up in inflation. Both rules assume that the underlying world is linear, which implies that the rule used is symmetric about the natural or NAIRU level of output.

In this paper we consider the performance of rules, and in particular 'optimal' rules, when the underlying relationship between inflation and the output gap may be non-linear. 
Schaling (1999) has explicitly examined the implications for the inflation-forecast targeting of a convex Phillips curve. Convexity in the inflation-output relationship implies that, under conditions of full employment, inflation seems to respond strongly to excess demand, while in deep recession it can be insensitive to the change in the level of activity. Several other recent studies (Laxton, Meredith and Rose, 1995; Turner, 1995; Clark, Laxton and Rose, 1996; Debelle and Laxton, 1997) argue that the level of economic activity has a nonlinear effect on inflation.

In this paper we add to this body of work by using a small empirical model of the US and UK economies to evaluate the performance of linear feedback rules when the underlying output-inflation relationship is non-linear ${ }^{1}$. Empirically an important difference between the US and the UK is that whereas real interest rates appear in the IS relationship for output, as prior theorising would suggest, for the UK nominal interest rates appear to matter. This means that in the US a shock to inflation has to change nominal interest rates by more than the shock in order to change real interest rates, while for the UK this is not necessary. This becomes especially important when there is a zero floor to nominal interest rates, because in the US the rise in real interest rates when inflation is negative depresses output more than in the UK.

We first demonstrate on the lines of Schaling (1999), that when there are non-linearities in the inflation-output relationship, the interest rate rule is similar to the Taylor rule but with state-contingent weights on the two disequilibrium terms which increase with the degree of non-linearity in the inflation-output relationship. We show that this nonlinearity in the interest rate response imparts a bias to the mean inflation rate when a linear feedback rule is used. Also adopting the skewness measure of Aizenman and Hausmann (1994) we show that when we adopt the modified hyperbola in Chadha et al (1992) for inflation, then this is positively skewed. Our simulation results confirm that in a non-linear setting for the US and UK, the adoption of a linear rule imparts a bias to the inflation rate. We also show that a piecewise linear or mixing feedback rule that provides an approximation to the non-linear rule of Schaling (1999) reduces the bias in the mean inflation rate and the positive skewness of inflation.

In the last section we examine the performance of linear and piecewise rules when a zero bound on nominal interest rate is operative. Summers (1991) emphasises that the drawback of price stability is in fact that the central bank is seriously constrained in its ability to offset adverse spending shocks because interest rate cannot turn negative, thus cushioning aggregate output. To establish the effect of the zero floor on nominal interest rate we perform our analysis simply switching off our feedback rule in the linear and piecewise setting, when nominal interest rate turns negative.

Our simulation results suggest that with a zero bound on interest rate output variability increases since the nominal interest rate cannot be further reduced in presence of adverse shocks.

\section{A Benchmark Model of Output and Inflation}

An increasingly common framework for organising the discussion of theoretical and empirical issues concerning monetary policy uses a simple IS relationship for deviations, in the short run, of output from trend (the output gap) combined with a Phillips curve and a loss function for the policymaker that provides a metric for the conduct of policy. A very general form of this model that encompasses a wide variety of theoretical perspectives and a linear and nonlinear Phillips curve is given below:

\footnotetext{
${ }^{1}$ It should be emphasised that in Phillip's original 1958 article he specifically considered a non-linear relationship between wage inflation and unemployment.
} 


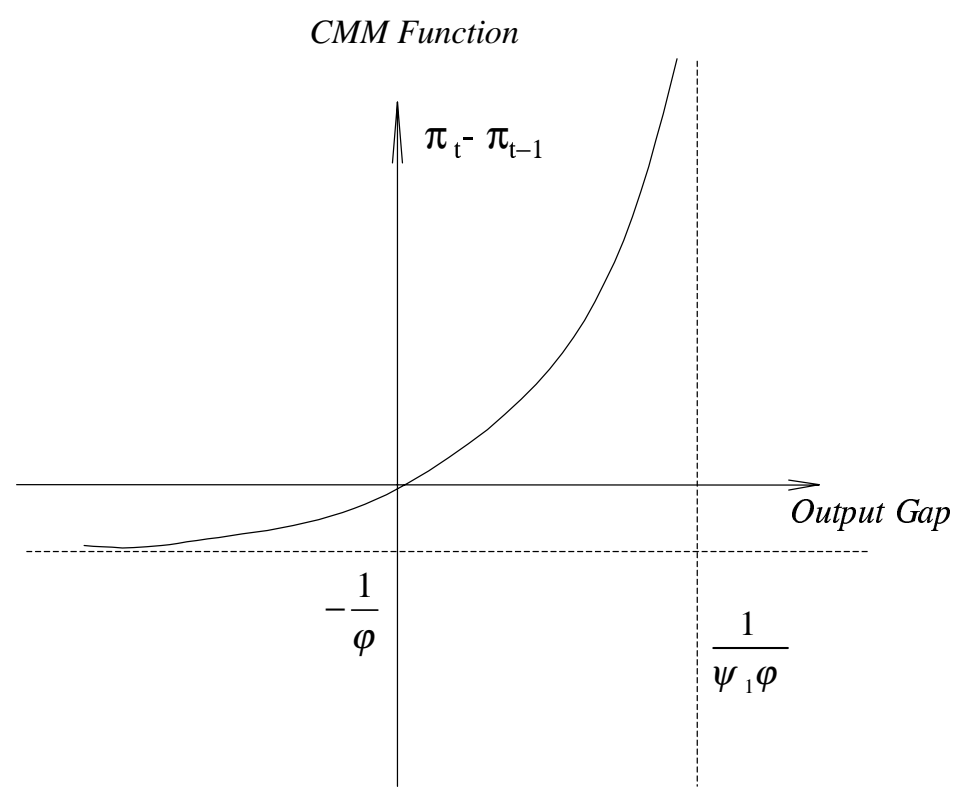

Figure 1: The CMM Inflation Function

$$
\begin{gathered}
y_{t+1}=\beta_{1} y_{t}+\beta_{2} E_{t+1} y_{t+2}-\left[d\left(\sum_{i=0}^{p} \alpha_{i}\left(i_{t-i}-E_{t+1} \pi_{t-i+1}\right)\right)+(1-d) \sum_{i=0}^{p} \alpha_{i} i_{t-i}\right]+v_{t+1} \\
\Delta \pi_{t+1}=\gamma_{1} \Delta \pi_{t}+\gamma_{2} E_{t+1} \Delta \pi_{t+2}+\sum_{i=-s}^{q} \frac{\psi_{i+s+1} y_{t+i}}{1-\psi_{i+s+1} \varphi y_{t+i}} \\
\min _{\left\{i_{t}\right\}} E_{t} \sum_{\tau=t}^{\infty} \delta^{\tau-t}\left\{\omega_{1}\left[\frac{\left(\pi_{\tau+2 \mid t}-\pi^{*}\right)^{2}}{2}\right]+\omega_{2}\left[\frac{\left(y_{\tau}\right)^{2}}{2}\right]\right\}
\end{gathered}
$$

$y$ is the output gap, $i$ is the short term nominal interest rate, $\pi$ is the inflation rate and $E$ the expectations operator; $v$ is an exogenous demand shock and $d$ is an indicator function which takes value one if the IS curve depends on the real interest rate and zero if it depends only on the nominal rate.

The relationship describing the inflation process is that proposed by Chadha et al (1992) and Laxton et al (1995). This modified hyperbola is shown in Figure 1.

Let us assume $\gamma_{1}=0$ and $\gamma_{2}=0$. The modified hyperbola can be written as:

$$
\Delta \pi_{t+1}=\frac{\psi_{1} y_{t}}{\left(1-\psi_{1} \varphi y_{t}\right)}
$$

There are a number of limiting cases: 


$$
\lim _{\varphi \rightarrow 0} \frac{\partial \pi}{\partial y}=\psi_{1}
$$

which is the linear trade-off between inflation and output.

$$
\lim _{y \rightarrow \frac{1}{\psi_{1} \varphi}} \frac{\partial \pi}{\partial y}=\infty
$$

where $\frac{1}{\psi_{1} \varphi}$ is the upper bound to the short run increase in output. While:

$$
\lim _{y \rightarrow-\infty} \frac{\partial \pi}{\partial y}=0
$$

At this lower limit, even though output is collapsing, inflation is completely unresponsive to output. It is clear from the first limiting condition that $\varphi$ captures the degree of curvature of the non-linear function. The third relationship in the benchmark model is the quadratic loss function usually employed in the literature ${ }^{2}$.

A variety of different versions of this framework are nested in the benchmark model. A dynamic general equilibrium approach to macroeconomics has been used, for example, by Goodfriend and King (1997) and McCallum (1999) in order to derive a forward looking version where $\varphi=0, \beta_{1}=0, \beta_{2}=1, \gamma_{1}=0, \gamma_{2}=1, s=-1, q=1, d=0$ and $p=0$. The current output gap depends on the real interest rate, now and in the future, through the expected output gap term. Inflation also depends on the current output gap and its future evolution. Alternatively, the backward looking approach of Taylor (1994), Svensson (1997) and Bean (1998) emphasises that, empirically, there are pure delays in the impact of interest rates on output, and output on inflation. In this case $\varphi=0, \beta_{2}=0, \gamma_{2}=0$, $\gamma_{1}=1$, and $q=-1$. This means that there is a pure delay of two years between a change in interest rates and it showing up in the rate of inflation. In both classes of model the solution to the policy problem, with an assumption about the formation of expectations, can be expressed as a feedback rule. This can be derived explicitly or written as a Taylor type rule ${ }^{3}$.

We now derive a feedback rule for the nominal interest rate from a simplified model nested in (1) where we assume $s=q=0, p=0, \gamma_{1}=0, \gamma_{2}=0$ and $\alpha_{i}=1$.

The model simplifies to:

$$
\begin{gathered}
y_{t+1}=\beta_{1} y_{t}-\left(d\left(i_{t}-\pi_{t}\right)+(1-d) i_{t}\right)+v_{t+1} \\
\Delta \pi_{t+1}=f(\bullet)=\frac{\psi_{1} y_{t}}{1-\psi_{1} \varphi y_{t}}
\end{gathered}
$$

where $\psi_{1}>0, \leq \varphi<1$ and $0<\beta_{1}<1$.

As in Svensson (1997) we assume that the interest rate in period $t$ affects inflation only starting in year $t+2$. Therefore the dynamic programming problem can be solved by assigning the interest rate in $t$ to the inflation rate two periods ahead. Following Schaling (1999) let $V\left(\pi_{t}, y_{t}\right)$ represent the value function. The minimization problem becomes:

$$
V\left(\pi_{t}, y_{t}\right)=\min _{\left\{i_{t}\right\}}\left\{E_{t}\left(q_{1}\left[\frac{\left(\pi_{\tau+2}-\pi^{*}\right)^{2}}{2}\right)+\delta E_{t}\left[V\left(\pi_{t}+f(\bullet), y_{t+1}\right)\right]\right\}\right.
$$

Expected inflation two years ahead is:

\footnotetext{
${ }^{2}$ For a recent discussion of the quadratic case see Chadha and Schellekens (1999).

${ }^{3}$ For a recent survey of this class of model see Clarida et al (1999).
} 


$$
E_{t} \pi_{t+2}=\pi^{*}-\frac{1}{\delta^{2} \psi_{1}} \frac{\omega_{2}}{\omega_{1}} y_{t}\left(1-\psi_{1} \varphi y_{t}\right)^{2}
$$

Therefore the inflation forecast two years ahead equals the inflation target plus a correction factor which depends upon the degree of non-linearity of the inflation-output relationship and on the relative weights placed upon the deviation of inflation and output from their target in the loss function. Considering the limiting value for expected inflation if the degree of non-linearity tends to zero we get:

$$
\lim _{\varphi \rightarrow 0} E_{t} \pi_{t+2}=\pi^{*}-\frac{1}{\delta^{2} \psi_{1}} \frac{\omega_{2}}{\omega_{1}} y_{t}
$$

which is the result in Svensson (1997). That is, the two year inflation forecast should equal the inflation target only if output equals the natural rate. Otherwise it should exceed the inflation target in proportion to how much output falls short of the natural level.

The feedback rule for the nominal interest rate, as in Schaling (1999), is given by:

$$
\left\{\begin{array}{llr}
i_{i}=\pi_{t}+r r^{*}+\frac{1}{\psi_{1}} \Psi\left(\pi_{t}-\pi^{*}\right)+\frac{1}{\psi_{1}} \Psi f(\bullet)+\beta_{1} y_{t} & \text { for } & d=1 \\
i_{i}=r r^{*}+\frac{1}{\psi_{1}} \Psi\left(\pi_{t}-\pi^{*}\right)+\frac{1}{\psi_{1}} \Psi f(\bullet)+\beta_{1} y_{t} & \text { for } & d=0
\end{array}\right\}
$$

where $\Psi=\left(\frac{1}{1-\varphi\left[\left(\pi_{t}-\pi^{*}\right)+f(\bullet)\right]}\right)$ and $r r^{*}$ is the real interest rate. The relationships in (10) are Taylor type rules with state-contingent weights.

Let us consider the rule when $d=1$. Since $\lim _{\varphi \rightarrow 0} \Psi=1$ and $\lim _{\varphi \rightarrow 0} f(\bullet)=\psi_{1} y_{t}$ the feedback rule (10) reduces to:

$$
i_{i}=\pi_{t}+r r^{*}+\frac{1}{\psi_{1}}\left(\pi_{t}-\pi^{*}\right)+\left(1+\beta_{1}\right) y_{t}
$$

which is the simple Taylor rule for the linear model where the nominal interest rate is set to equal inflation plus the real rate plus two disequilibrium terms. One of the disequilibrium terms is the discrepancy of actual inflation from the target; the other disequilibrium term is the output gap, $y$.

On the other hand, if there are non-linearities in the inflation-output relationship, the Taylor rule becomes state-contingent and the weights on the two disequilibrium terms increase with the degree of non-linearity in the inflation-output relationship.

How does this non-linearity affect the distribution of inflation realisations in a stochastic environment?

A simple measure of the degree of asymmetry of the inflation response is given by the skewness of the distribution:

$$
\mu_{3}=\frac{E\left[\left\{\left(\pi_{t}-\pi_{t}^{*}\right)-E\left[\left(\pi_{t}-\pi_{t}^{*}\right)\right]\right\}^{3}\right]}{\left[E\left\{\left(\left(\pi_{t}-\pi_{t}^{*}\right)-E\left[\pi_{t}-\pi_{t}^{*}\right]\right)^{2}\right\}\right]^{1.5}}
$$

The level of inflation derived from (10) is given by:

$$
\left\{\begin{array}{rlrl}
\pi_{t} & =\pi_{t}^{*}+\frac{1}{\beta_{1} \varphi y_{t}-\varphi\left(r_{t}-r r^{*}\right)-\frac{1}{\psi_{1}}}\left[(\varphi f(\bullet)-1)\left(\beta_{1} y_{t}-\left(r_{t}-r r^{*}\right)\right)+\frac{1}{\psi_{1}} f(\bullet)\right] & \text { for } & d=1 \\
\pi_{t}=\pi_{t}^{*}+\frac{1}{\beta_{1} \varphi y_{t}-\varphi\left(i_{t}-r r^{*}\right)-\frac{1}{\psi_{1}}}\left[(\varphi f(\bullet)-1)\left(\beta_{1} y_{t}-\left(i_{t}-r r^{*}\right)\right)+\frac{1}{\psi_{1}} f(\bullet)\right] & \text { for } & d=0
\end{array}\right\}
$$


where $r_{t}=i_{t}-\pi_{t}$ denotes the real interest rate. We denote $\pi_{t}=h(x)$ and:

$$
\left\{\begin{array}{lll}
x=\left(r_{t}, y_{t}\right) & \text { for } & d=1 \\
x=\left(i_{t}, y_{t}\right) & \text { for } & d=0
\end{array}\right\}
$$

the vector $x$ having mean $x^{*}$ and standard deviation $\sigma_{x}$. The relationship (13) shows that the deviation of inflation from target is a non linear function of output, $y$ and the interest rate. Therefore can use a second order Taylor approximation around $x_{0}$ :

$$
h(x) \cong h\left(x^{*}\right)+\left(x-x^{*}\right) h^{\prime}\left(x^{*}\right)+0.5\left(x-x^{*}\right)^{2} h^{\prime \prime}\left(x^{*}\right)
$$

which implies

$$
E[h(x)] \cong h\left(x^{*}\right)+0.5\left(x-x^{*}\right)^{2} h^{\prime \prime}\left(x^{*}\right)
$$

Hence it is possible to demonstrate (Aizenman and Hausmann, 1994) that:

$$
\mu_{3}=\frac{\left.1.5\left[E\left(x-x^{*}\right)^{4}-\left(\sigma_{x}\right)^{4}\right]\right] h^{\prime \prime}\left(x^{*}\right)}{\left|h^{\prime}\left(x_{0}\right)\right|\left(\sigma_{x}\right)^{2}}
$$

from which we conclude that:

$$
\operatorname{sign}\left(\mu_{3}\right)=\operatorname{sign} h^{\prime \prime}\left(x^{*}\right)
$$

We can derive the effect of the interest rate and output on inflation as:

$$
\left\{\begin{array}{c|cccc}
\frac{\partial^{2} \pi_{t}}{\partial r_{t}^{2}} & \left.\right|_{x^{*}}\left(d r_{t}\right)^{2}+\left.\frac{\partial^{2} \pi_{t}}{\partial y_{t}^{2}}\right|_{x^{*}}\left(d y_{t}\right)^{2} & \text { for } & d=1 \\
\left.\frac{\partial^{2} \pi_{t}}{\partial i_{t}^{2}}\right|_{x^{*}}\left(d i_{t}\right)^{2}+\left.\frac{\partial^{2} \pi_{t}}{\partial y_{t}^{2}}\right|_{x^{*}}\left(d y_{t}\right)^{2} & \text { for } & d=0
\end{array}\right\}
$$

Evaluating the relationships in (19) at $x^{*}\left(r r^{*}, y^{*}\right)$ with output in equilibrium being equal to its natural level $\left(y^{*}=0\right)$ we get the condition:

$$
\begin{gathered}
\left.\frac{\partial^{2} \pi_{t}}{\partial i_{t}^{2}}\right|_{x^{*}}=\left.\frac{\partial^{2} \pi_{t}}{\partial r_{t}^{2}}\right|_{x^{*}}=2 \varphi \psi_{1}^{2}>0 \\
\left.\frac{\partial^{2} \pi_{t}}{\partial y_{t}^{2}}\right|_{x^{*}}=2 \varphi \psi_{1}^{2}\left(2-\beta_{1}\left(1-\beta_{1}\right)\right)>0
\end{gathered}
$$

Therefore:

$$
\operatorname{sign}\left(\mu_{3}\right)=\left\{\begin{array}{lll}
\operatorname{sign}\left(2 \varphi \psi_{1}^{2}\left((d i)^{2}+\left(2-\beta_{1}\left(1-\beta_{1}\right)\right)(d y)^{2}\right)\right) & \text { for } & d=1 \\
\operatorname{sign}\left(2 \varphi \psi_{1}^{2}\left((d r)^{2}+\left(2-\beta_{1}\left(1-\beta_{1}\right)\right)(d y)^{2}\right)\right) & \text { for } & d=0
\end{array}\right\}>0
$$

So the sign of the skewness depends on the sign of $2 \varphi \psi_{1}^{2}$, which is always positive. The positive skewness can also be inferred from Figure 1 where the hyperbolic function is drawn. At low levels of output the effect on inflation dies away, while for high levels of output the effect of the output gap on inflation increases, so it is not surprising that more of the mass of the distribution of inflation outcomes is concentrated to the right. 


\section{Some Empirical Evidence for the UK and US}

In this section we turn to some empirical evidence of non-linearities and asymmetries in the Phillips curve for the US and the UK. We use quarterly data from 1966 to 1997 and we estimate equation (1b) setting either $d=1$ when the real interest rate is used in the IS relationship for the US or $d=0$ when the nominal interest rate is used for the UK. In Table 1 we report estimates of a number of different functional forms for the Phillips curve. In column one we show a linear version of the Phillips curve. This is a very parsimonious formulation. In line with other evidence, current deviations of output from trend did not have a significant effect on inflation, but the lagged output gap is significant ${ }^{4}$.

In column two we report a kinked functional form, with the discontinuity occurring at the zero output gap. This single threshold model can be written as:

$$
\Delta \pi_{t}=\gamma_{1} \Delta \pi_{t-1}+\theta_{1}^{+} y_{t-1}^{+}+\theta_{1}^{-} y_{t-1}^{-}
$$

$y_{t-1}^{+}$and $y_{t-1}^{-}$refer to observations of the output gap lying above and below zero, respectively. Finally in column three we report estimates of a two-threshold, or piecewise linear formulation. The general form of this model can be written as:

$$
\Delta \pi_{t}=\gamma_{1} \Delta \pi_{t-1}+\theta^{1} y_{t-1} I_{1}+\theta^{2} y_{t-1} I_{2}+\theta^{3} y_{t-1} I_{3}
$$

The terms $I_{i}$ with $i=1,2,3$ are defined as follows:

$$
\begin{aligned}
& I_{1}=\left\{1 \quad \text { for } y_{t-1}<\phi_{1}, 0 \text { otherwise }\right\} \\
& I_{2}=\left\{1 \quad \text { for } \phi_{1}<y_{t-1}<\phi_{2}, 0 \text { otherwise }\right\} \\
& I_{3}=\left\{1 \quad \text { for } y_{t-1}>\phi_{2}, 0 \text { otherwise }\right\}
\end{aligned}
$$

where $\phi_{1}$ and $\phi_{2}$ are the threshold values. Continuity of this function requires the imposition of the following constraints:

$$
\begin{aligned}
\theta^{1} \phi_{1} & =\theta^{2} \phi_{1} \\
\theta^{2} \phi_{2} & =\theta^{3} \phi_{2}
\end{aligned}
$$

Expanding (23) and substituting in the continuity constraints yields:

$\pi_{t}=\pi_{t-1}+\gamma_{1} \Delta \pi_{t-1}+\phi_{1}\left(\theta^{2}-\theta^{1}\right) I_{1}+\phi_{2}\left(\theta^{2}-\theta^{3}\right) I_{3}+\theta^{1} y_{t-1} I_{1}+\theta^{2} y_{t-1} I_{2}+\theta^{3} y_{t-1} I_{3}+u_{t}$

Equation (26) embodies a piecewise linear function with a kink at the point where the output gap begins to exert upward (above $\phi_{2}$ ) and downward (below $\phi_{1}$ ) pressure on inflation. It can be thought of as an approximation of any hyperbolic functional form, which is convex whenever $\theta_{3} \geqq \theta_{2} \geqq \theta_{1}$, concave whenever $\theta_{3} \leqq \theta_{2} \leqq \theta_{1}$, and concaveconvex if $\theta_{3} \leqq \theta_{2} \geqq \theta_{1}$. Its advantage is that it is possible to test for asymmetry in a very direct way.

When the economy is overheated, above $\phi_{2}$, the Phillips curve is steeper, with a given increase in output having a larger effect on inflation. Below $\phi_{1}$, when the economy is depressed, the effect of output on inflation weakens. This can also be thought of as a piecewise linear approximation to a sigmoid. A special case of these piecewise functional forms is the kinked function of Laxton, Rose and Tetlow (1993), where the kink is at the

\footnotetext{
${ }^{4}$ We estimate the output gap using a Hodrick-Prescott filter
} 


\begin{tabular}{|c|c|c|c|c|c|c|}
\hline & & & One $\mathrm{t}$ & eshold & Two tl & esholds \\
\hline & US & UK & US & UK & US & UK \\
\hline$\gamma_{1}$ & 0.472 & 0.345 & 0.470 & 0.346 & 0.463 & 0.332 \\
\hline & $(6.28)$ & $(4.91)$ & $(6.24)$ & $(4.90)$ & $(6.45)$ & $(4.75)$ \\
\hline$\theta_{1}$ & $\begin{array}{l}0.077 \\
(379)\end{array}$ & 0.225 & & & & \\
\hline & & & & & & \\
\hline$\theta_{1}^{+}$ & & & 0.065 & 0.212 & & \\
\hline & & & $(2.25)$ & (2.71) & & \\
\hline$\theta_{1}^{-}$ & & & 0.088 & 0.23 & & \\
\hline & & & (3.31) & (3.35) & & \\
\hline$\theta^{1}$ & & & & & 0.20 & 0.58 \\
\hline & & & & & $(3.00)$ & $(1.25)$ \\
\hline$\theta^{2}$ & & & & & 0.02 & 0.15 \\
\hline & & & & & $(0.78)$ & (2.17) \\
\hline$\theta^{3}$ & & & & & 0.46 & 0.75 \\
\hline & & & & & $(3.97)$ & $(2.31)$ \\
\hline$\phi_{1}$ & & & & & -0.02 & -0.02 \\
\hline$\phi_{2}$ & & & & & 0.02 & 0.03 \\
\hline$R^{2}$ & 0.42 & 0.26 & 0.42 & 0.26 & 0.48 & 0.26 \\
\hline SEE & 0.003494 & 0.010605 & 0.003503 & 0.010637 & 0.003146 & 0.010691 \\
\hline $\operatorname{LM}(2)$ & 0.182 & 0.453 & 0.213 & 0.512 & 0.182 & 0.231 \\
\hline $\mathrm{ARCH}(1)$ & 0.474 & 0.213 & 0.552 & 0.223 & 0.474 & 0.260 \\
\hline
\end{tabular}

Table 1: Phillips Curve Estimates 
point where the output gap is zero. This is similar to the non-symmetric error correction model of Granger and Lee $(1989)^{5}$.

The third column in Table 1 contains the two threshold specification of the Phillips curve. As in Filardo (1998) using quarterly data for the US, we find very significant non-linearities. In the weak economy regime, the slope is 0.2 . In the balanced economy regime the slope is essentially flat whereas in the overheated-economy regime the slope is 0.46, more than twice as steep as in the weak-economy regime. For the UK we find that a concave-convex relationship fits more or less as well as the linear model, though a test of this against the null of a linear model gave a $\chi^{2}=2.99(p=0.223)$. Since a J-test against the alternatives is inconclusive, we can reject neither specification. As in the case of the US, in the balanced regime the slope of the Phillips curve is positive but very close to zero and substantially less in the weak than over-heated regime and with an almost flat slope in the balanced economy, suggesting that inflation does not respond to the output gap in this regime. In the overheated regime the inflation-output relationship is $30 \%$ steeper than in the weak regime, suggesting that inflation is much more sensitive in this region.

\subsection{Modelling Output}

In this section we augment the relationship for inflation with an empirical equation for the output gap.

For the US we use a backward-looking version of the IS curve, which contains the lagged real interest rate on the right-hand side. In fact as shown in previous research (Roberts, 1995; McCallum and Nelson, 1998), this specification for the IS curve seems to fit the data better.

As in Fuhrer and Estrella (2000) we use the federal funds rate for the nominal interest rate. Inflation is defined in terms of the chain-weighted GDP deflator, rather than the CPI.

The IS equation is the following:

$$
\begin{aligned}
& \qquad \begin{array}{cc}
y_{t, \text { usa }}=0.0018-0.075\left(i_{t-1}-\pi_{t-1}\right)+0.8975 y_{t-1} \\
(-2.45)
\end{array} \\
& R^{2}=0.76 \quad S E E=0.008 \quad L M(2)=6.71 \\
& \text { Sample Period: } 1966: 4-1998: 2
\end{aligned}
$$

Because this is a model of the output gap we estimated the equation so that the long run output gap is zero by imposing the restriction that the intercept offsets of approximately 2.5 percent the average real interest rate over the sample period.

Note, that while the IS model for the US is in terms of the real interest rate, it proved impossible with UK data to find a significant effect except when the nominal interest rate was used. This is what we report and use in the simulations later in the paper. Following Bean (1998) we allow the interest rate to have a different effect on output depending upon whether a fixed exchange rate regime is in operation or not.

\footnotetext{
${ }^{5}$ See Escribano and Pfann (1998) for an extended discussion of non-symmetric error correction models.
} 


$$
\begin{aligned}
& y_{t, u k}=-0.0004 D_{1}+0.011 D_{2}-0.013 D_{1}\left(i_{t-3}+i_{t-4}\right) \underset{(-0.07)}{(-3.67)} \\
& R^{2}=0.72 \quad S E E=0.009 D_{2}\left(i_{t-3}+i_{t-4}\right)+0.79 y_{t-1} \\
& (15.55) \\
& L M(2)=0.251 \quad A R C H(1)=0.276
\end{aligned}
$$

Sample Period: 1969:1 - 1997:4

$D_{1}$ and $D_{2}$ are dummy variables; $D_{1}$ takes the value 1 from prior to 1972 and 0 thereafter while $D_{2}$ takes the value 0 prior to 1972 and 1 thereafter.

\section{The Optimal Control Rule}

To solve for the optimal control rule we use the standard LQG (Linear Quadratic Gaussian) approach. First we define a loss function for the monetary authorities in terms of a state variable $z$ :

$$
L_{t}=\frac{1}{2} \sum_{t=0}^{n}\left(z_{t, j}-z_{t, j}^{d}\right)^{\prime} Q_{i}\left(z_{t, j}-z_{t, j}^{d}\right)
$$

for $j=U S, U K$ where:

$$
\begin{aligned}
& z_{t, u s}=\left[\begin{array}{l}
y_{t} \\
\pi_{t} \\
\pi_{t-1} \\
i_{t} \\
i_{t-1} \\
\Delta i_{t-1}
\end{array}\right] \quad z_{t, u s}^{d}=\left[\begin{array}{l}
0 \\
0 \\
0 \\
0 \\
0 \\
0
\end{array}\right] \quad Q_{u s}=\left[\begin{array}{lllll}
\omega_{1} & 0 & 0 & 0 & 0 \\
0 & \omega_{2} & 0 & 0 & 0 \\
0 & 0 & 0 & 0 & 0 \\
0 & 0 & 0 & 0 & 0 \\
0 & 0 & 0 & 0 & 0 \\
0 & 0 & 0 & 0 & \omega_{3}
\end{array}\right] \\
& z_{t, u k}=\left[\begin{array}{l}
y_{t} \\
\pi_{t} \\
\pi_{t-1} \\
i_{t} \\
i_{t-1} \\
i_{t-2} \\
i_{t-3} \\
\Delta i_{t-1}
\end{array}\right] \quad z_{t, u k}^{d}=\left[\begin{array}{l}
0 \\
0 \\
0 \\
0 \\
0 \\
0 \\
0
\end{array}\right] \quad Q_{u k}=\left[\begin{array}{llllllll}
\omega_{1} & 0 & 0 & 0 & 0 & 0 & 0 & 0 \\
0 & \omega_{2} & 0 & 0 & 0 & 0 & 0 & 0 \\
0 & 0 & 0 & 0 & 0 & 0 & 0 & 0 \\
0 & 0 & 0 & 0 & 0 & 0 & 0 & 0 \\
0 & 0 & 0 & 0 & 0 & 0 & 0 & 0 \\
0 & 0 & 0 & 0 & 0 & 0 & 0 & 0 \\
0 & 0 & 0 & 0 & 0 & 0 & 0 & 0 \\
0 & 0 & 0 & 0 & 0 & 0 & 0 & \omega_{3}
\end{array}\right]
\end{aligned}
$$

The vector of state variables $z$ is chosen to make the model easy to write in state-space form. The state-space form of a model relates the current state to its one period lag, the current value of the control variable and a set of (possibly time varying) intercept terms as shown in (30):

$$
z_{t, j}=A_{j} z_{t-1, j}+B_{j} i_{t, j}+e_{t, j}
$$

where: 


$$
\begin{gathered}
A_{u s}=\left[\begin{array}{llllll}
\beta_{1} & -\alpha_{1} & 0 & \alpha_{1} & 0 & 0 \\
\theta_{1} & 1+\gamma & -\gamma & 0 & 0 & 0 \\
0 & 1 & 0 & 0 & 0 & 0 \\
0 & 0 & 0 & 0 & 0 & 0 \\
0 & 0 & 0 & 1 & 0 & 0 \\
0 & 0 & 0 & -1 & 0 & 0
\end{array}\right], \quad B_{u s}=\left[\begin{array}{l}
0 \\
0 \\
0 \\
1 \\
0 \\
1
\end{array}\right] \\
A_{u k}=\left[\begin{array}{llllllll}
\beta_{1} & 0 & 0 & 0 & 0 & \alpha_{3} & \alpha_{3} & 0 \\
\theta_{1} & 1+\gamma & -\gamma & 0 & 0 & 0 & 0 & 0 \\
0 & 1 & 0 & 0 & 0 & 0 & 0 & 0 \\
0 & 0 & 0 & 0 & 0 & 0 & 0 & 0 \\
0 & 0 & 0 & 1 & 0 & 0 & 0 & 0 \\
0 & 0 & 0 & 0 & 1 & 0 & 0 & 0 \\
0 & 0 & 0 & 0 & 0 & 1 & 0 & 0 \\
0 & 0 & 0 & -1 & 0 & 0 & 0 & 0
\end{array}\right], \quad B_{u k}=\left[\begin{array}{l}
0 \\
0 \\
0 \\
1 \\
0 \\
0 \\
0 \\
1
\end{array}\right]
\end{gathered}
$$

Note that the state vector is defined in order to include the control variable $i$, and its first difference $\Delta i$, as part of the state. This enables us to place penalties on either deviations of the interest rate from some target value or on changes in interest rates. Such penalties are proved to be extremely important for the nature of the control solution.

The general solution for this form of the control problem can be written as:

$$
i_{t, j}=K_{t, j} z_{t-1, j}+k_{t, j}
$$

where $K_{t, j}(t=1, . ., T)$ are a sequence of feedback control matrices and $k_{t, j}(t=$ $1, \ldots, T)$ represents what is known as the tracking gain in the control literature. These are solved for recursively by first solving the period $T$ problem to obtain a solution for $i_{T, j}$ conditional on $i_{T-1, j}$. This is used to write a value function for period $T$ which depends on $i_{T-1, j}$ which in turn forms part of the objective function for the period $T-1$ problem. Using this procedure, along with the terminal conditions $H_{T, j}=Q_{j}$ and $k_{T, j}=h_{T, j}=$ $Q_{j} z_{T, j}^{d}$ we can solve for the sequence of feedback control matrices and tracking gains as:

$$
\begin{aligned}
K_{T, j} & =-\left(B_{j}^{\prime} H_{T, j} B_{j}\right)^{-1}\left(B_{j}^{\prime} H_{T, j} A_{j}\right) \\
k_{T, j} & =-\left(B_{j}^{\prime} H_{T, j} B_{j}\right)^{-1} B_{j}^{\prime}\left(H_{T, j} e_{T, j}-h_{T, j}\right) \\
H_{T-1, j} & =Q_{j}+\left(A_{j}+B_{j} K_{T, j}\right)^{\prime} H_{T, j}\left(A_{j}+B_{j} K_{T, j}\right) \\
h_{T-1, j} & =k_{T-1, j}+\left(A_{j}+B_{j} K_{T, j}\right)^{\prime}\left(h_{T, j}-H_{T, j} e_{T, j}\right)
\end{aligned}
$$

These are the well-known discrete time Ricatti equations. Since we have assumed that the $B_{j}, A_{j}$ and $Q_{j}$ matrices are constant, it is also possible to show that the feedback control matrices should converge to a constant matrix for some $t<t^{*}$. The tracking gain will only converge if both the targets in the loss function and the intercepts in the state-space representation of the model are constant.

\section{Some Model Simulations}

In this section we turn to a stochastic analysis of the small model with and without the non-linear Phillips curve. Our intention is to evaluate how well an 'optimal' rule derived on the assumption that the underlying Phillips curve is linear, behaves when in fact the Phillips curve is non-linear. 


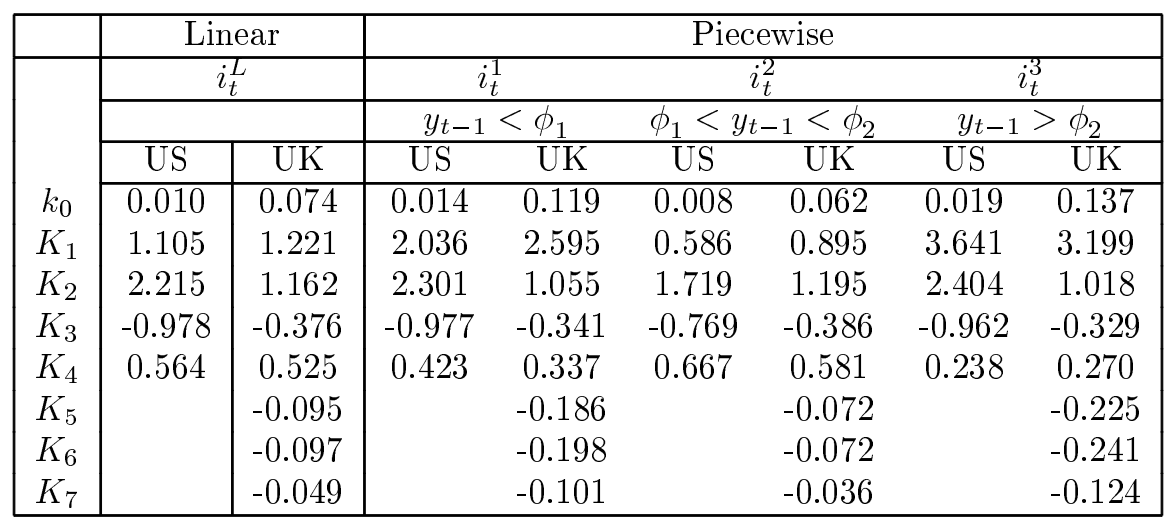

Table 2: Feedback Rule Coefficients

The optimal rule is the equilibrium solution for the optimal control problem. This is obtained by iterating the Ricatti equations until the feedback control rule converges. The solution takes the form ${ }^{6}$ :

$$
i_{t, u s}=k_{0}+K_{1} y_{t-1}+K_{2} \pi_{t-1}+K_{3} \pi_{t-2}+K_{4} i_{t-1}
$$

$$
i_{t, u k}=k_{0}+K_{1} y_{t-1}+K_{2} \pi_{t-1}+K_{3} \pi_{t-2}+K_{4} i_{t-1}+K_{5} i_{t-2}+K_{6} i_{t-3}+K_{7} i_{t-4}
$$

In order to make the rules comparable we impose equal weights of 1 on output deviation, inflation deviations and nominal interest rate deviations from a target of 0 percent, both for US and UK.

The first column of Table 2 shows the optimal linear feedback rule for the US and the UK. Given the weights used, the feedback on output is very strong in both countries and a shock to output brings a countervailing change in interest rates larger than the magnitude of the shock. In response to an inflation shock in the US nominal interest rates are changed by more than the size of the shock in order to change the interest rate in the right direction. In the UK because nominal interest rates do the work it is not necessary to change nominal interest rates by as much as the shock.

In Table 3 we show the steady state results of 2000 stochastic simulations $^{7}$ of the model. In conducting our stochastic simulations we allow the interest rate to respond to lagged stochastic disturbances to the inflation and output equation over the simulation period, using the feedback control coefficients determined through dynamic programming. Column 1 shows the results of simulating the linear model using the feedback rule calculated above. The error covariances we use are those provided by the estimated equations ${ }^{8}$.

This generates a steady state solution for the linear model with the output gap and inflation at zero. This provides the benchmark for the subsequent simulations. As it would be expected, given that the random shocks are drawn from a normal distribution,

\footnotetext{
${ }^{6}$ The extra terms in the UK rule reflect the extra lagged interest rate terms in the IS curve.

${ }^{7}$ The stochastic simulations use the antithetics suggested by Bianchi, C., Calzolari, G., Corsi P. (1978)

${ }^{8}$ Sgherri and Wallis(1997) have argued in another context that using the covariances from the full sample will exaggerate the width of the bands around the target for inflation. The current UK policy regime has a band of $\pm 1 \%$ about a target of $2.5 \%$. Sgherri and Wallis (1997) show that if the covariances of the errors for the $1990 \mathrm{~s}$ are used the probability of staying within the range is $90 \%$. However, our interest here is different. We are concerned to evaluate the effects of nonlinearities (and zero nominal interest rate floors). If the shocks are too small we may fail to excite those regions of the nonlinear space that we are interested in.
} 
the distribution of output and inflation outcomes are also normally distributed about the target. However, given the seeds that we have used for the stochastic simulations the standard error on the nominal interest rate is very large. If the target for inflation was indeed $2.5 \%$ for the UK and around $2 \%$ for the US, this would imply negative interest rates. For the present let us assume that the target inflation rate is sufficiently high for this not to be a problem. We shall return to this issue later.

The question we want to ask is how well does a rule calculated on the assumption that the Phillips curve is linear, perform in a world in which the Phillips curve actually takes on the piecewise linear form of equation (26).

Table 3 column 2 shows that using the linear rule in a non-linear world imparts a bias to the steady state inflation rate which is positive for the US and negative for the UK, which may be caused by the difference in the width of the output regimes in the two economies. For the UK the higher Phillips curve slope in the overheated regime is activated only when the positive shock on output exceeds $3 \%$, whereas for the US the upper threshold is reached when the shock on output is $2 \%$. We can see this visually in Figures 2 and 3. Here we have plotted kernel density estimates of the steady state distribution of inflation, output and the interest rate. The dotted line represents the linear model with the linear feedback rule, $i_{t}^{L}$, reported in the first two columns of Table 2 . The solid line is the distribution when the Phillips curve is non-linear but the feedback rule linear. For inflation the mass of the distribution is shifted to the right and for the US there is a positive skewness which suggest a high degree of non-linearity for the inflation-output relationship. Significant positive skewness in US inflation is also mirrored in significant negative skewness in output and positive skewness in the interest rate. There is also evidence of significant kurtosis in both the US and the UK in inflation and interest rates. The kurtosis can be traced to the weaker effect that the output gap has on inflation in the middle region. Therefore, the optimal rule has more difficulty keeping inflation in a narrow band about the mean. This is reflected in both higher variance and more kurtosis with fatter tails.

As we have shown, Schaling (1999) has derived a particular feedback rule which contains the hyperbolic function. What we do here, is to approximate this function with a piecewise linear feedback rule. Given the slope coefficients reported in column 3 of Table 1 , for the different segments of the convex function, we calculated the three rules $i_{t}^{1}, i_{t}^{2}$, $i_{t}^{3}$. Table 2 shows the value of the feedback rule in the different regimes for the US and UK. Note that in the middle regime, where inflation is almost unresponsive to output, the feedback on output deviations is moderate, whereas it becomes extremely responsive in the over-heated regime, where the slope of the Phillips curve is higher, in particular for the US where we have found the slope in the upper output regime is more than twice as steep as it is in the lowest regime. If there is an inflation shock the nominal interest rate responds more in the US than in the UK in order to offset the inflation shock and increase the real interest rate.

The mixing feedback rule is then defined as follows:

$$
i_{t}^{m}=i_{t}^{1} I_{1}+i_{t}^{2} I_{1}+i_{t}^{3} I_{3}
$$

where as before:

$$
\begin{array}{llll}
I_{1}=\{1 & \text { for } & y_{t-1}<\phi_{1}, 0 & \text { otherwise }\} \\
I_{2}=\{1 & \text { for } & \phi_{1}<y_{t-1}<\phi_{2}, 0 & \text { otherwise }\} \\
I_{3}=\{1 & \text { for } & y_{t-1}>\phi_{2}, 0 & \text { otherwise }\}
\end{array}
$$




\begin{tabular}{|c|c|c|c|c|c|c|}
\hline \multicolumn{7}{|c|}{ Number of Solutions 2000 - Simulation Period 1998:4-2006:4 } \\
\hline & \multicolumn{2}{|c|}{$\mathrm{LM} / \mathrm{LFR}$} & \multicolumn{2}{|c|}{ NLM/LFR } & \multicolumn{2}{|c|}{ NLM/PLFR } \\
\hline & US & UK & $\overline{\mathrm{US}}$ & UK & US & UK \\
\hline \multicolumn{7}{|l|}{ Means of Series } \\
\hline Deviation of inflation from target (\%) & 0 & 0 & 1.9 & -2.56 & 0.92 & -0.73 \\
\hline Deviation of output from target (\%) & 0 & 0 & -0.81 & 0.56 & -0.15 & 0.32 \\
\hline \multicolumn{7}{|l|}{ Standard Deviation } \\
\hline Inflation(\%) & 3.88 & 7.73 & 6.44 & 11.46 & 10.25 & 9.99 \\
\hline Growth(\%) & 3.28 & 3.50 & 3.95 & 4.09 & 2.62 & 3.68 \\
\hline Interest Rate(\%) & 11.43 & 9.78 & 18.51 & 15.02 & 16.94 & 14.94 \\
\hline \multicolumn{7}{|l|}{ Skewness } \\
\hline Inflation & 0.00 & 0.00 & 0.88 & -0.017 & -0.007 & 0.062 \\
\hline Growth & 0.00 & 0.00 & -0.23 & 0.094 & -0.231 & 0.030 \\
\hline Interest Rate & 0.00 & 0.00 & 0.73 & 0.035 & 0.044 & 0.131 \\
\hline \multicolumn{7}{|l|}{ Kurtosis } \\
\hline Inflation & 3.17 & 3.08 & 5.75 & 4.96 & 2.93 & 3.70 \\
\hline Growth & 2.86 & 2.82 & 3.25 & 3.01 & 2.50 & 2.92 \\
\hline Interest Rate & 3.19 & 3.24 & 5.47 & 5.37 & 3.08 & 2.77 \\
\hline \multicolumn{7}{|l|}{ Bowman-Shenton $\left(\chi^{2}(2)\right)$} \\
\hline Inflation & 2.63 & 0.60 & 892.72 & 323.09 & 0.321 & 42.24 \\
\hline Growth & 1.57 & 2.43 & 24.53 & 3.0177 & 38.23 & 0.776 \\
\hline Interest Rate & 3.15 & 5.15 & 692.28 & 470.14 & 1.27 & 9.843 \\
\hline \multicolumn{7}{|l|}{ 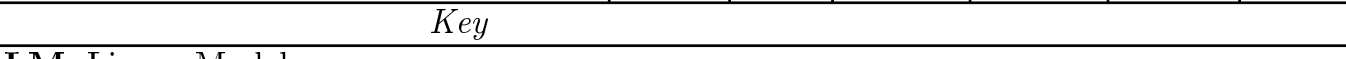 } \\
\hline $\begin{array}{l}\text { LM: Linear Model } \\
\text { LFR: Linear Feedback Rule } \\
\text { NLM: Non-Linear Model } \\
\text { PLFR: Piecewise Linear Feedback }\end{array}$ & & & & & & \\
\hline
\end{tabular}

Table 3: Steady State Results from the Stochastic Simulations

At each threshold there is a discrete switch to the linear rule appropriate to the differently sloped Phillips curve.

In Table 3 column 2 and Figures 4 and 5 we show that the mixing feedback rule in the US reduces the inflation bias arising from the non-linear Phillips curve, as well as the skewness in inflation, but with continuing kurtosis in output. For the UK, the piecewise rule reduces the inflation bias, lowers the standard deviation in inflation, output and interest rates, and eliminates the kurtosis, but at the expense of increasing the skewness of the interest rate.

In conclusion the differences between the two countries can be traced to: $(i)$ the different slopes of the nonlinear Phillips curve; (ii) the differential impact of nominal and real interest rates on output; (iii) the different thresholds.

\subsection{The Zero Floor on Interest Rates}

We have already drawn attention to the wide standard error bands overlapping with a negative region for the nominal interest rate. In this section we analyse the implications of a zero bound on interest rates for the conduct of the monetary policy for both the linear and nonlinear environments. Recent research (Fuhrer and Madigan, 1997) has stressed that when the zero bound on nominal interest rate is operative this create a constraint on the central bank's ability to offset adverse spending shocks since interest rates cannot 
UK: LM/LFR-NLM/LFR: Kernel Distribution
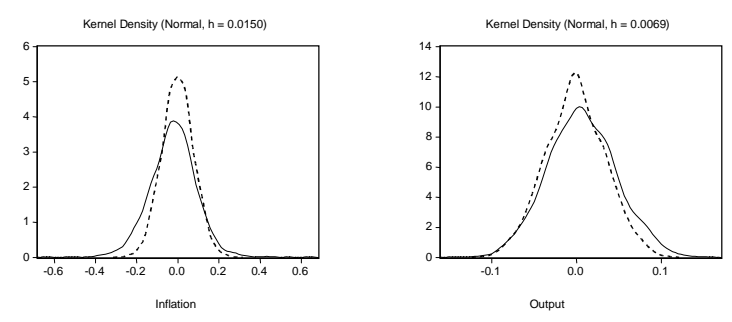

Output

LM/LFR

NLM/LFR

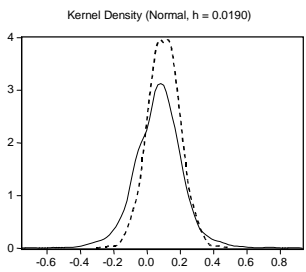

Interest Rate

Figure 2: UK-Kernel Distribution: comparison between the Linear Model with a Linear Feedback Rule (LM/LFR) and the Non Linear Model with a Linear Feedback Rule (NLM/LFR).

USA: LM/LFR-NLM/LFR: Kernel Distribution
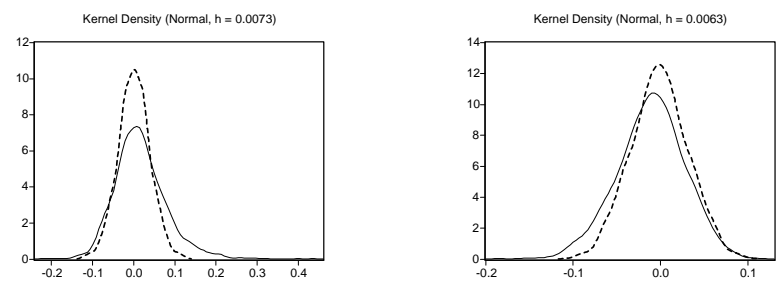

Inflation

Kernel Density (Normal, $\mathrm{h}=0.0224$ )

Output
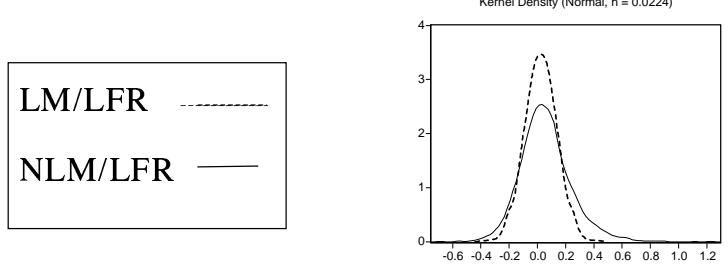

Interest Rates

Figure 3: USA-Kernel Distribution: comparison between the Linear Model with a Linear Feedback Rule (LM/LFR) and the Non Linear Model with a Linear Feedback Rule (NLM/LFR). 
UK: LM/LFR-NLM/PLFR: Kernel Distribution
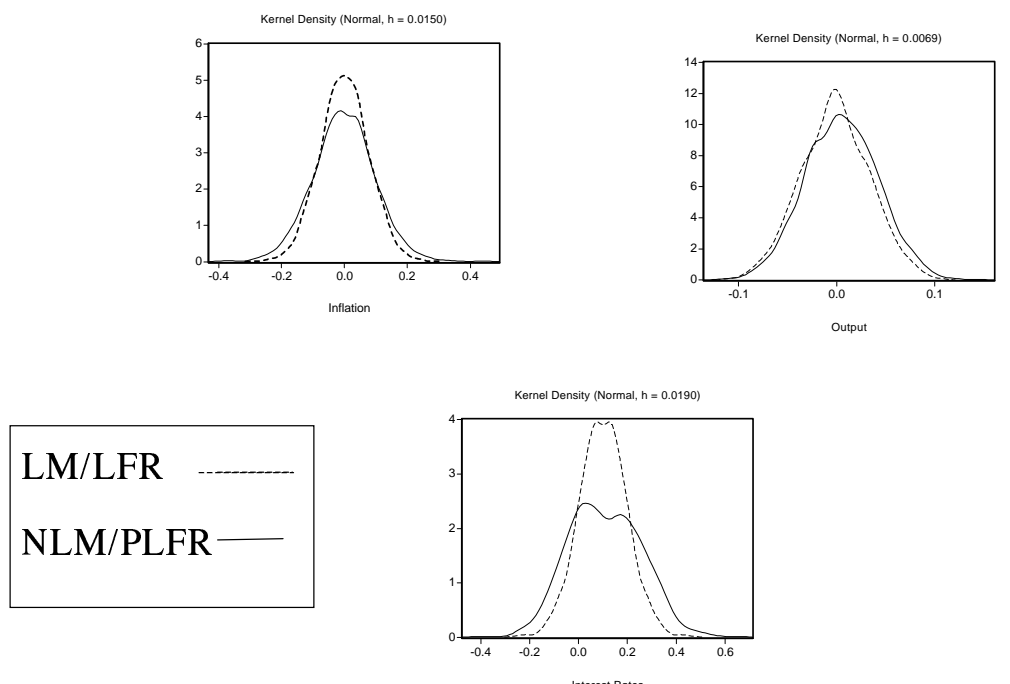

Figure 4: UK-Kernel Distribution: comparison between the Linear Model with a Linear Feedback Rule (LM/LFR) and the Non Linear Model with Piecewise Linear Feedback Rule (NLM/PLFR).

USA: LM/LFR-NLM/PLFR: Kernel Distribution
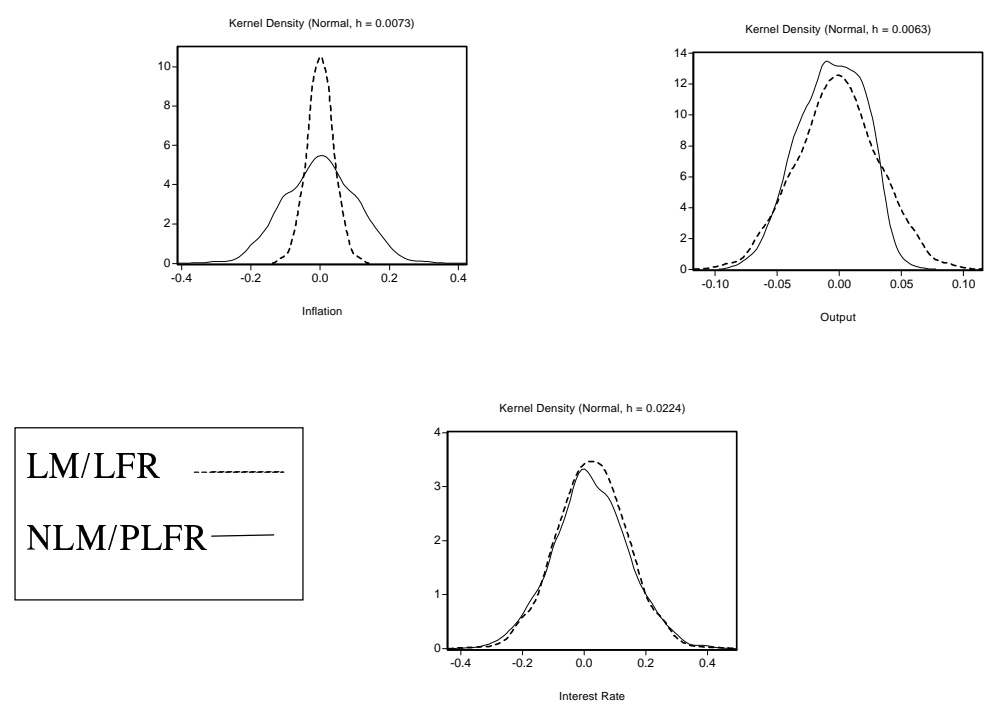

Figure 5: USA-Kernel Distribution: comparison between the Linear Model with a Linear Feedback Rule (LM/LFR) and the Non Linear Model with Piecewise Linear Feedback Rule (NLM/PLFR). 
be negative ${ }^{9}$.

To implement the zero floor rule we switch off the optimal rule when the interest rate hits the floor. This is a rather crude method but it does have the advantage of simplicity and allows us to evaluate in a straightforward way what the implications of the zero floor might be. This is equivalent to assuming a nominal interest rate response of the form:

$$
i_{t, i}^{z}=\max \left\{i_{t}, 0\right\}
$$

for $i=\mathrm{US}, \mathrm{UK}$ where $i^{z}$ represents the linear optimal rule for the interest rate with a zero floor whereas for the mixing piecewise rule we have:

$$
i_{t, i}^{z, m}=\max \left\{i_{t}^{m}, 0\right\}
$$

where $i^{z, m}$ represents the optimal mixing rule when the zero bound on nominal interest rates applies.

Let us take the linear case first. Table 4 column one suggests that when the rule is operative inflation is negatively skewed for both the US and the UK. Moreover, there is a negative output bias for the US and a positive output bias for the UK. This arises because of the real interest rate effect on output which is absent in the UK model. This is in line with previous findings (Wolman, 1998) which show that when the zero bound on interest rates is operative there is a larger fall in output at the zero inflation target. The differential interest rate effect also shows up in more skewness and kurtosis for the US.

When we consider the nonlinear case, shown in Figures 8 and 9, we find that the distortions to the distribution of inflation and output that are triggered by the interest rate floor are compounded by the nonlinearity. There is more mean bias, skewness and kurtosis for both countries. When we adopt the mixing feedback rule, there is little improvement. The mean bias actually increases in the US.

\footnotetext{
${ }^{9}$ For a recent discussion of the precise meaning of this and how some institutional arrangements might be designed to allow negative nominal interest rates on fiat money see Buiter and Panigirtzoglou (2000).
} 


\begin{tabular}{|c|c|c|c|c|c|c|}
\hline \multicolumn{7}{|c|}{ Number of Solutions 2000 - Simulation Period 1998:4-2006:4 } \\
\hline & \multicolumn{2}{|c|}{ LM/LFR } & \multicolumn{2}{|c|}{ NLM/LFR } & \multicolumn{2}{|c|}{ NLM/PLFR } \\
\hline & $\overline{\mathrm{US}}$ & UK & US & UK & US & UK \\
\hline \multicolumn{7}{|l|}{ Means of Series } \\
\hline Deviation of inflation from target (\%) & -1.2 & -0.1 & -0.7 & -3.8 & -2.2 & -1.0 \\
\hline Deviation of output from target (\%) & -1.2 & 0 & -1.8 & 0.2 & -2.1 & 1.3 \\
\hline \multicolumn{7}{|l|}{ Standard Deviation } \\
\hline Inflation(\%) & 6.21 & 8.32 & 14.5 & 12.72 & 18.45 & 13.38 \\
\hline Growth(\%) & 4.25 & 3.60 & 5.85 & 3.70 & 5.44 & 3.58 \\
\hline \multicolumn{7}{|l|}{ Skewness } \\
\hline Inflation & -0.71 & -0.19 & 0.93 & -0.22 & 0.02 & 0.19 \\
\hline Growth & 0.01 & 0.018 & 0.45 & -0.12 & 0.12 & -0.19 \\
\hline \multicolumn{7}{|l|}{ Kurtosis } \\
\hline Inflation & 4.51 & 3.34 & 11.9 & 4.52 & 6.75 & 4.54 \\
\hline Growth & 3.52 & 2.89 & 6.04 & 3.01 & 7.08 & 2.99 \\
\hline \multicolumn{7}{|l|}{ Bowman-Shenton $\left(\chi^{2}(2)\right)$} \\
\hline Inflation & 360.4 & 23.06 & $>1000$ & 209.7 & $>1000$ & 210.56 \\
\hline Growth & 22.83 & 0.98 & 845.02 & 5.6 & $>1000$ & 12.74 \\
\hline \multicolumn{7}{|l|}{ Key } \\
\hline \multicolumn{7}{|l|}{$\begin{array}{l}\text { LM: Linear Model } \\
\text { LFR: Linear Feedback Rule } \\
\text { NLM: Non-Linear Model } \\
\text { PLFR: Piecewise Linear Feedback Rule }\end{array}$} \\
\hline
\end{tabular}

Table 4: Steady State Results with an Interest Rate Floor

\section{Conclusions}

We have explored a number of issues concerned with the design of monetary policy in a world in which the Phillips curve might be significantly nonlinear and in which there is a zero bound to the nominal interest rate. We have investigated not only the behaviour of the first two moments of the distribution of outcomes but also skewness and kurtosis.

Using small empirical models of the US and the UK we have been able to demonstrate with stochastic simulation techniques that a mixing rule which involves piecewise linear feedback rules corresponding to differently sloped parts of the Phillips curve, is very successful in correcting the mean biases that are induced by the nonlinearity and restoring much of the normal distribution of outcomes in the US, at least for inflation.

We have deliberately carried out the analysis over a wide range of stochastic outcomes in order to excite those regions in which the nonlinearity is important. It may be that this has little relevance for contemporary policymaking, when recent history has delivered relatively small shocks to inflation and output, and the monetary regimes that have been in place in the US for the last decade, and in the UK for the last 8 years, have been very successful in keeping inflation low and stable and the volatility of output at historically low levels. Within the bands that current policy has delivered, the relationship between output and inflation is linear. So the results that we have for regions well outside recent experience may be of esoteric interest. The only qualification to this comforting conclusion is the effect that the interest rate floor has on policy effectiveness even in the linear case. This suggests that some thought should be given to how to design rules that in certain states of the world avoid the floor without unacceptable output costs. This is also of some relevance for the debate on the merits of gradualism versus cold turkey responses 
UK: LM/LFR-NLM/LFR: Kernel Distribution
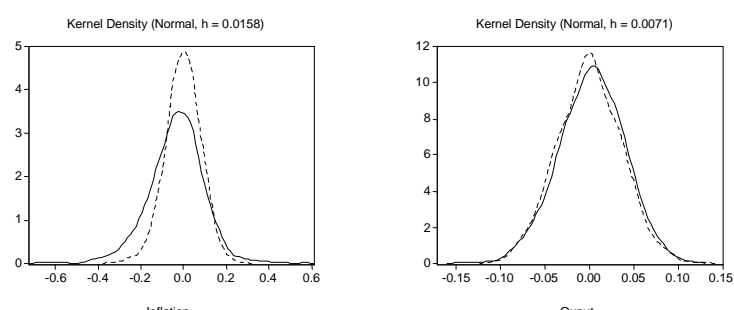

Ouput

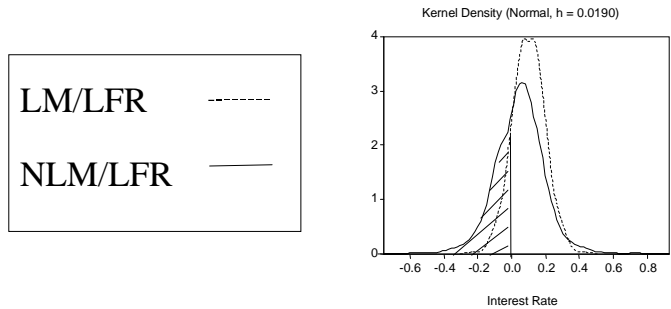

Figure 6: UK- Zero Floor on the interest rate. Kernel Distribution: comparison between the Linear Model with a Linear Feedback Rule (LM/LFR) and the Non Linear Model with a Linear Feedback Rule (NLM/LFR).

USA: LM/LFR-NLM/LFR: Kernel Distribution
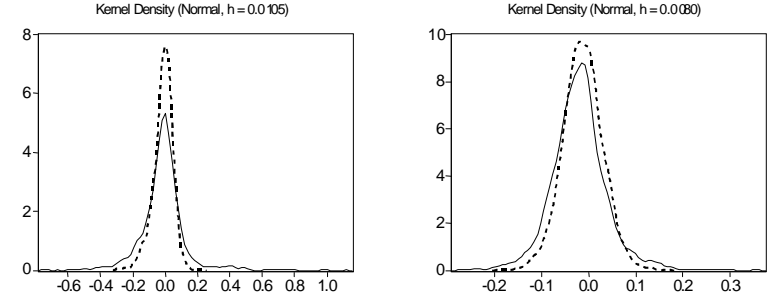

Inflation

Kerne Density (Normal, $\mathrm{h}=0.0224) \quad$ Output
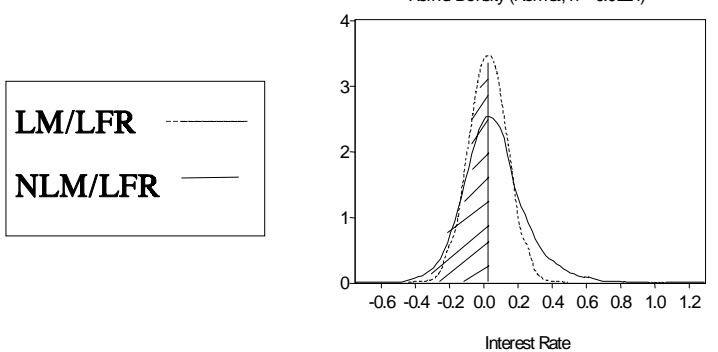

Figure 7: USA- Zero Floor on the interest rate. Kernel Distribution: comparison between the Linear Model with a Linear Feedback Rule (LM/LFR) and the Non Linear Model with a Linear Feedback Rule (NLM/LFR). 
UK: LMLFR-NLMPLFR: KernelDistribution
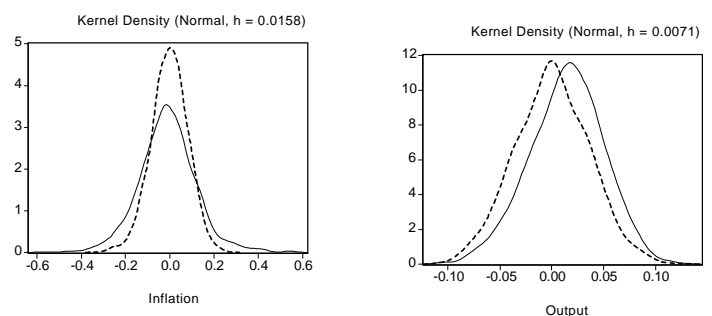

Intlation

Kenel Density (Nomala, $\mathrm{h}=0.0190$
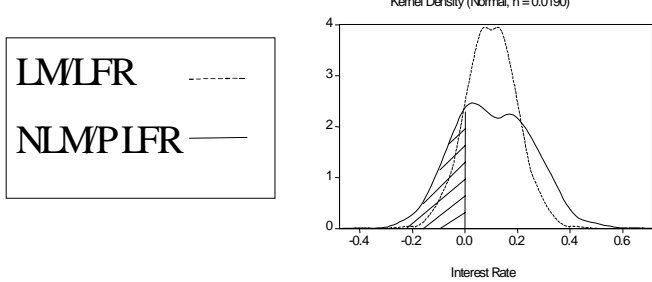

Figure 8: UK-Zero Floor on the interest rate. Kernel Distribution: comparison between the Linear Model with a Linear Feedback Rule (LM/LFR) and the Non Linear Model with Piecewise Linear Feedback Rule (NLM/PLFR).

\section{USA: LMLFR-NLMPLFR: Kernel Distribution}
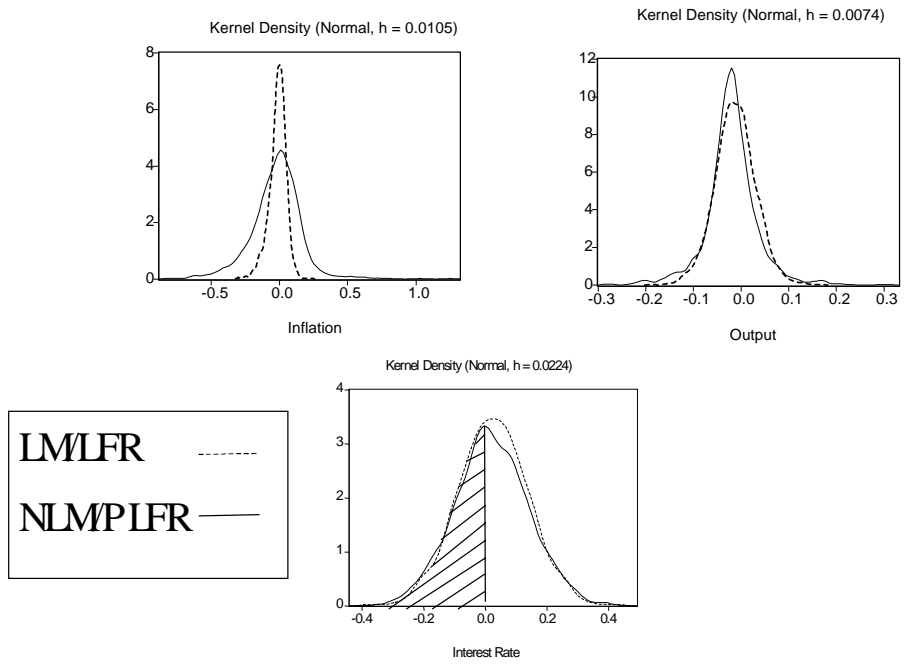

Figure 9: USA-Zero Floor on the interest rate. Kernel Distribution: comparison between the Linear Model with a Linear Feedback Rule (LM/LFR) and the Non Linear Model with Piecewise Linear Feedback Rule (NLM/PLFR). 
to shock. A large shock that temporarily moved the economy outside the comfort zone, may require swift and decisive action in order to bring the economy back under control before the non-linear processes have a chance to take effect.

\section{References}

[1] Aizenman, J. and R. Hausmann (1994), Why is Inflation Skewed? A Debt and Volatility Story, NBER working paper 4837.

[2] Bean, C. (1998), The New UK Monetary Arrangements: A View from the Literature, Economic Journal, November, pp.1795-1809.

[3] Bianchi, C., Calzolari, G., Corsi P. (1978), A Program for Stochastic Simulation of Econometric Models, Econometrica; 46(1), January, pages 235-36.

[4] Bowman, K.O. and Shenton, L.R. (1975), Omnibus test contours for departures from normality based on $\sqrt{ } b_{1}$ and $\mathrm{b}_{2}$, Biometrica, 62, 243-50.

[5] Buiter W.H and Panigirtzoglou, N (2000), Liquidity traps: how to avoid them and how to escape them, Bank of England Mimeo.

[6] Chadha, B, Masson, P and Meredith, G (1992), Models of Inflation and the Costs of Disinflation, IMF Staff Papers, Vol 39, No 2, June, pages 395-431.

[7] Chadha, J. and P. Schellekens (1999). "Monetary Policy Loss Functions - two cheers for the quadratic", Bank of England Working Paper no 101.

[8] Clark, P., Laxton, D. and Rose, D. (1995), Capacity Constraints, Inflation and the Transmission Mechanism: Forward-Looking Versus Myopic Policy Rules, IMF Working Paper, $95 / 75$.

[9] Debelle, G and Laxton, D (1997), Is the Phillips Curve Really a Curve? Some Evidence for Canada, the United Kingdom and the United States, IMF Staff Papers, Vol 44, No 2.

[10] Filardo, A. J. (1998), New Evidence on the Output Cost of Fighting Inflation, Federal Reserve Bank of Kansas City Economic Review.

[11] Estrella A. and J. Fuhrer (2000), Are Deep Parameters Stable? The Lucas Critique as an Empirical Hypothesis, paper presented at the ADRES Conference on the Econometrics of Policy Evaluation, Paris, January 10-12.

[12] Fuhrer, J. C. and B. F. Madigan (1997), Monetary Policy when Interest Rates are Bounded at Zero, The Review of Economics and Statistics.

[13] Granger, C.W.J. and T.H. Lee, (1989), Investigation of Production, Sales and Inventory Relationships using Multicointegration and Non-Symmetric Error Correction Models, Journal of Applied Econometrics, Vol. 4, pp. 145-159.

[14] Laxton, D., Meredith, G., Rose, D. (1995), Asymmetric Effects of Economic Activity on Inflation: Evidence and Policy Implications, IMF Staff Papers, 42(2), pages 344-74.

[15] Laxton, D., Rose, D., and Tetlow, R. (1993), Monetary Policy, Uncertainty and the Presumption of Linearity, Bank of Canada Technical Report 63, August.

[16] McCallum, B.T. and E. Nelson (1998), Operational Policy Rules in an Optimizing IS-LM Model, prepared for the joint CEPR/San Francisco Fed conference. 
[17] Roberts, J. (1995), New Keynesian Economics and the Phillips Curve, Journal of Money Credit and Banking, 37(4), 975-984.

[18] Schaling, E, (1999), The Non-Linear Phillips Curve and Inflation Forecast Targeting, Bank of England Working Paper series.

[19] Sgherri ,S. and Wallis, K.F. (1997), Inflation target bands and the Bank of England's fairly substantial lump of inflation uncertainty, ESRC Macroeconomic Modelling Bureau Discussion Paper No.49,October.

[20] Summers, L. J. (1991), How Should Long-Term Monetary Policy be Determined?, Journal of Money, Credit and Banking 23:3, 625-631.

[21] Svensson, Lars E.O. (1997), Inflation Forecast Targeting: Implementing and Monitoring Inflation Targets, European Economic Review, 41, 1111-1146.

[22] Taylor, John B. (1994), The Inflation/Output Variability Trade-off Revisited, Goals, guidelines, and constraints facing monetary policymakers: Proceedings of a conference held at North Falmouth, Massachusetts, June. Conference Series, no. 38. Boston: Federal Reserve Bank of Boston, 1994, pages 21-38.

[23] Turner, D (1995) Speed Limit and Asymmetric Inflation Effects from the Output Gap in the Major Seven Economies, OECD-Economic-Studies, 0(24), pp. 57-87.

[24] Wolman A. L. (1998), Staggered Price Setting and the Zero Bound on Nominal Interest Rates, Federal Reserve Bank of Richmond Economic Quarterly, 84/4. 